\title{
Cellule $\mathbf{R}_{1}$ et musculature des Filaires; analyse ultrastructurale (1)
}

\author{
par G. PETIT \\ Laboratoire de Zoologie-Vers, associé au C.N.R.S., \\ Muséum national d'Histoire naturelle, 43, rue Cuvier, F 75231 Paris Cedex 05.
}

RESUME. L'étude ultrastructurale de l'ontogénèse d'une Filaire, Dipetalonema (Molinema) dessetae, confirme l'hypothèse de Bain (1970), selon laquelle la cellule $\mathrm{R}_{1}$ intervient dans la formation de la musculature de la Filaire adulte.

L'analyse anatomique du fourreau épithélio-musculaire, les jonctions entre les divers types de cellules, l'existence de cellules intermédiaires entre les cellules embryonnaires issues de $R_{1}$ et les cellules musculaires, montrent que les cellules-filles de $R_{1}$, disposées en 4 files longitudinales, donnent naissance successivement, du $5^{\mathrm{e}}$ au $13^{\mathrm{e}}$ jour, à une dizaine de files de cellules musculaires, dans chaque champ submédian.

La musculature primordiale de la microfilaire, constituée d'une file de cellules musculaires par champ, n'est pas de nature ectodermique, comme il avait été supposé, mais mésenchymateuse.

Au contact des cellules issues de la $\mathrm{R}_{1}$, elle subit une légère dédifférenciation (environ 5 rangées de myofilaments, au lieu de 10-20 chez la microfilaire et la $\mathrm{L}_{3}$ ), puis son évolution s'aligne sur celle des cellules musculaires néoformées.

La cellule $\mathrm{R}_{1}$, dont l'existence et la destinée paraissent constantes chez tous les Nématodes Phasmidiens complètement hétéroxènes, présente donc une certaine analogie avec les disques imaginaux des Insectes holométaboles.

\section{$R_{1}$ cell and musculature of Filariae; ultrastructural analysis.}

SUMMARY. Ultrastructural study of ontogenesis of a filaria, Dipetalonema (M.) dessetea, confirms Bain's (1970) hypothesis, following which the $R_{1}$ cell plays a role in the elaboration of the musculature of the adult filaria.

(1) Ce travail a pu être effectué grâce à une subvention de l'Organisation Mondiale de la Santé. Accepté le 23 mai 1980. 
Anatomical observations related to the hypodermis and musculature of the larva (distribution of the cells, nature of their junctions, presence of intermediate cells between embryonic cells derived from $R_{1}$ cell and muscle cells) show that the cells derived from $R_{1}$, which form four longitudinal files, produced successively, from 5 th to 13 th day, about ten files of muscle cells in each submedian field.

The primary musculature of the microfilaria, which corresponds to a single file of muscular cells in each field, is not ectodermic, as supposed before, but mesenchimatic. At the vicinity of $R_{1}$ daughter cells, this musculature is slightly dedifferenciated (about 5 files of myofilaments instead of 10-20 in the microfilaria and the $L_{3}$ ) ; then its evolution becomes identical to this of new formed muscle cells.

The presence as well as the evolution of the $R_{1}$ cell seem to be identical in every totally heteroxenous Phasmidian Nematodes; both suggest analogy with the imaginal discs of the holometabolic Insects.

\section{Introduction}

La microfilaire est un jeune stade I en semi-diapause, qui possède quelques cellules remarquables par leur grande taille : la cellule excrétrice et les 4 cellules $R$ (ou $G$ ).

Alors qu'il est établi que les cellules $R_{2}, R_{3}$ et $R_{4}$ donnent les 3 glandes rectales de la Filaire (Schacher et Khalil, 1968 ; Bain, 1970 et 1972 ; Laurence et Simpson, 1971), le rôle de la cellule $R_{1}$ reste controversé.

Bain (1970) observe que la cellule $R_{1}$ est située entre le bourgeon rectal ectodermique et le bourgeon intestinal endodermique, et qu'elle subit une série de divisions pour former 4 files longitudinales de cellules, situées dans les 4 axes submédians de la larve. Ces deux faits l'amènent à supposer que la cellule $\mathrm{R}_{1}$ est une initiale mésenchymateuse, qui engendre la musculature de la Filaire et elle pose comme hypothèse que les cellules contractiles de la microfilaire sont ectodermiques.

Cette dernière hypothèse n'est pas vérifiée, car les analyses ultrastructurales (Kozek, 1971 ; Mac Laren, 1972 ; Johnston et Stehbens, 1973 ; Kanagasuntheram et coll., 1974 ; Laurence et Simpson, 1974) montrent que les 4 files longitudinales de cellules muscu. laires de la microfilaire reposent sur l'ectoderme et n'en font pas partie.

L'origine de la musculature semble donc complexe, non seulement chez les Filaires, mais chez d'autres Nématodes hétéroxènes pour lesquels l'ontogénèse et les divisions de la cellule $\mathrm{R}_{1}$ sont identiques à ce qui a été décrit par Bain :

Diplotriaenoidea : Bain et Vaucher, 1973 ; Aproctoidea : Quentin et coll., 1976 ; Spiruroidea : Bain, 1972 ; Quentin et Poinar, 1973 ; Camallanoidea : Vaucher et Bain, 1973 ; Seuratoidea : Quentin et Poinar, 1973 ; Subuluroidea : Quentin et Poinar, 1973.

Nous nous proposons donc de suivre l'évolution de la cellule $\mathrm{R}_{1}$ en microscopie électronique, en prenant comme exemple la Filaire de laboratoire Dipetalonema (Molinema) dessetae Bain, 1973. 
MATÉriel ET TEChNioues.

L'entretien de D. (M.) dessetae chez Aedes aegypti, (souche Gkep) permet d'obtenir de fortes infestations, avec un développement synchrone et rapide des larves en 15 jours, à $27^{\circ}$.

Le tissu adipeux des Aedes parasités est fixé et contrasté pour la microscopie électronique suivant les techniques décrites précédemment (Petit et Spitalier-Kaveh, 1979).

L'analyse morphologique des larves est faite sur des coupes transversales sériées, effectuées d'arrière en avant, à partir du pore anal.

\section{I. - MISE EN PLACE DES FILES LONGITUDINALES DE CELLULES EMBRYON- NAIRES ISSUES DE LA CELLULE $R_{1}$.}

La microscopie électronique confirme les observations faites en microscopie optique.

Au $5^{\mathrm{e}}$ jour, la cellule $R_{1}$ s'est divisée 2 fois dans le sens transversal et a donné naissance à 4 cellules occupant chacune un des champs latéromédians, en avant des cellules rectales ( $f$ ig. $1 \mathrm{~B}, 2 \mathrm{~B}$ et fig. 3). Chacune de ces 4 initiales donne naissance, par divisions successives dans le sens longitudinal, à 4 files qui progressent d'arrière en avant et atteignent la région œsophagienne dès le $6^{\mathrm{e}}$ jour. Il apparaît que ces files longitudinales ont une situation un peu variable, car elles sont tantôt accolées aux cordes médianes, tantôt aux cordes latérales; elles restent toujours séparées de ces formations ectodermiques par une lame basale.

Au cours du développement, la cellule $R_{1}$ et ses descendants sont faciles à identifier par leur aspect ultrastructural : gros noyau à chromatine complètement dispersée et nucléole bien individualisé; cytoplasme avec ribosomes souvent sous forme de polysomes, granules de glycogène, corps de Golgi ; il y a peu de réticulum endoplasmique.

Ce sont des cellules indifférenciées, qui ne forment jamais d'attaches fixes (hemidesmosomes ou desmosomes) ni avec l'ectoderme, ni avec les cellules musculaires; elles conservent l'aspect de cellules embryonnaires.

\section{II. - FORMATion DES Cellules MUSCUlaires ISSUES DE LA LIGNEE $R_{1}$.}

Plusieurs images histologiques indiquent clairement que les cellules musculaires sont formées les unes après les autres par les divisions transversales successives des files des cellules $R_{1}$. La preuve de ce phénomène est apportée par les observations suivantes :

a. Dans chaque champ submédian, les cellules filles de $\mathrm{R}_{1}$ sont étroitement accolées aux cellules musculaires (fig. $2 \mathrm{~B}, \mathrm{C}, \mathrm{D}, \mathrm{E}$ et fig. 3, 4, 5 et 6 ) pour former un ensemble bien individualisé, nettement séparé de l'ectoderme par une lame basale. 
b. Dans quelques cas, il existe entre la cellule de type $\mathrm{R}_{1}$ et les cellules musculaires une cellule d'aspect intermédiaire (fig. 2 E et fig. 6) : comme les cellules musculaires voisines, elle est accrochée à l'hypoderme par des hémidesmosomes et elle possède des microtubules à sa base ; mais, comme la cellule fille de $R_{1}$, elle est toujours dépourvue de myofilaments.

La formation des cellules musculaires commence à partir du $5^{\mathrm{e}}$ jour $(2$ cellules musculaires par champ submédian) (fig. 2 B, fig. 3); elle est terminée chez la larve en mue II âgée de 13 jours (fig. 7), qui a environ 11 cellules par champ submédian, toutes musculaires, la lignée $\mathrm{R}_{1}$ n'étant plus morphologiquement distincte.

\section{III. - EVOLUTION DE LA MUSCULATURE PRIMITIVE DE LA MICROFILAIRE.}

Comme il a été montré chez les microfilaires de différentes espèces, la musculature est constituée de 4 files longitudinales de cellules musculaires.

Sur une coupe du $5^{\mathrm{e}}$ jour située juste en avant du rectum, nous voyons la cellule de la lignée $R_{1}\left(R_{1}\right)$ accolée à 2 cellules musculaires $\left(M_{1}\right)$ et $\left(M_{2}\right)$ (fig. 2 B et fig. 3 ). La cellule $\mathrm{M}_{1}$ a un cytoplasme clair ; la cellule $\mathrm{M}_{2}$, bien que déjà pourvue de myofilaments, a un cytoplasme identique à celui de la $R_{1}$. Nous pensons donc que la cellule claire correspond à la cellule musculaire primordiale et que la $\mathrm{M}_{2}$ vient d'apparaître par division de la $\mathrm{R}_{1}$.

Sur une coupe correspondante du $6^{\mathrm{e}}$ jour, nous observons au contraire une cellule $\mathrm{R}_{1}$ englobant 2 cellules musculaires identiques entre elles (fig. $2 \mathrm{C}$ et fig. 4).

Il semble donc que les cellules musculaires primordiales restent en place et soient simplement remaniées au contact des cellules nouvelles-nées. Aucune image de lyse de la musculature primordiale et aucune image de phagocytose n’a jamais été rencontrée.

L'ultrastructure des musoles de la microfilaire montre l'existence de 10 à 15 rangées de myofilaments épais ; lors du développement de la Filaire ,les cellules musculaires, aussi bien la primordiale dédifférenciée que les néoformées, n’ont plus que 5 rangées environ de myofilaments.

$\mathrm{Au} 13^{\mathrm{e}}$ jour, les cellules musculaires ont développé leur partie contractile et possèdent une vingtaine de rangées de myofilaments épais.

\section{Conclusion}

La musculature de la Filaire Dipetalonema (Molinema) dessetae et, semble$\mathrm{t}$-il, celle de tous les Nématodes Phasmidiens complètement hétéroxènes (1), est mise en place en deux temps successifs.

(1) Nous entendons par là, les Nématodes dont le cycle évolutif, du début de la $\mathrm{L}_{1}$ jusqu'à la $\mathrm{L}_{3}$, s'effectue chez un hôte intermédiaire. 
L'essentiel de la musculature (environ 10 files de cellules musculaires sur 11, dans chacun des quatre champs) provient des cellules issues de la division de la $R_{1}$.

La musculature primordiale de la microfilaire, réduite à une file de cellules musculaires par champ, ne dégénère pas. Elle subit une légère dédifférenciation et son développement s'aligne ensuite sur celui des cellules musculaires néoformées.

La cellule $R_{1}$, la plus grande des cellules existant chez une microfilaire, qui reste à l'état quiescent jusqu'au $3^{\mathrm{e}}$ jour du développement chez le vecteur, évoque donc, à une échelle réduite, les cellules embryonnaires des disques imaginaux qui interviennent dans la métamorphose des Insectes holométaboles.

\section{Symboles des légendes des figures}

$\mathrm{CD}$, corde dorsale ; $\mathrm{Ci}$, cellule intermédiaire; $\mathrm{CL}$, corde latérale; $\mathrm{D}$, desmosome ; Exc, cellule excrétrice; $\mathrm{Gn}$, cellule nerveuse ; $\mathrm{H}$, Hèmocèle ; $h$, hypoderme ; HD, hemidesmosome , I, intestin; LB, lame basale; $\mathbf{M}$, cellule musculaire; $\mathbf{M}_{1}$, cellule musculaire primordiale ; $\mathrm{M}_{2}$, cellule musculaire néoformée ; $\mathrm{m}$, mue ; $\mathrm{mt}$, microtubule ; my, myofilaments ; $\mathrm{N}$, noyau ; $n$, filet nerveux; $\mathbb{E}$, œsophage; $\operatorname{Pr}$, prérectum; $R_{1}$, cellule $R_{1}$ ou de la lignée $R_{1}$; $T A$, tissu adipeux du moustique.

\section{Bibliographie}

Bain O.: La cellule $\mathrm{R}_{1}$ des microfilaires (Nematoda) initiale du mésenchyme. Ann. Parasitol. Hum. Comp., 1970, 45, 227-235.

Bain O.: Recherches sur la morphogénèse des Filaires chez l'hôte intermédiaire. Ann. Parasitol. Hum. Comp., 1972, 47, 251-303.

Bain O.: Une nouvelle Filaire de Rongeur sud-américain, Dipetalonema dessetae n. sp. (Nematoda, Filarioidea). Bull. Mus. Nat. Hist. Nat., Paris, $3^{e}$ sér., 1973, 116, 309-316.

Bain O.: Développement larvaire de Dipetalonema dessetae, Filaire de Rongeur entretenue au laboratoire. Ann. Parasitol. Hum. Comp., 1974, 49, 451-466.

Bain O., Vaucher $\mathrm{Cl}$. : Développement larvaire de Diplotriaena tridens (Nematoda: Filarioidea) chez Locusta migratoria. Ann. Parasitol. Hum. Comp., 1973, 48, 81-89.

Johnston M.R. L., Stehbens W.E.: Ultrastructural studies on the microfilaria of Cardianema sp. Alicata, 1933 (Nematoda; Onchocercidae). Int. J. Parasitol., 1973, 3, 243-250.

Kanagasuntheram R., Singh M., Ho B.C., Chan H. L. : Some ultrastructural observations on the microfilaria of Breinlia sergenti - the excretory complex, rectal cells and anal vesicle. Int. J. Parasitol., 1974, 4, 7-15.

Kozek W. J. : Ultrastructure of the microfilaria of Dirofilaria immitis. J. Parasitol., 1971, 57, $1052-$ 1067.

Laurence B.R., Simpson M.G.: The Microfilaria of Brugia: a first stage Nematoda Larva. J. Helminth., 1971, 45, 23-40.

Laurence B. R., Simpson M. G.: The ultrastructure of the microfilaria of Brugia (Nematoda: Filarioidea). Int. J. Parasitol., 1974, 4, 523-536.

Mac Laren D. J.: Ultrastructural studies on microfilariae (Nematoda: Filarioidea). Parasitology, $1972,65,317-332$.

Petit G., Spitalier-Kaveh H. : La Filaire Dipetalonema dessetae chez Aedes aegypti; étude du tissu adipeux parasité. Ann. Parasitol. Hum. Comp., 1979, 54, 81-92. 
Quentin J.C., Poinar G. O.: Comparative study of the larval development of some heterexenous subulurid and spirurid Nematodes. Int. J. Parasitol., 1973, 3, 809-827.

Quentin J. C., Troncy P.M., Barre N.: Aprocta cylindrica Linstow, 1883, Filaire ovipare parasite d'oiseaux Plocéides au Tchad. Morphogénèse larvaire du Nématode. Ann. Parasitol. Hum. Comp., 1976, 51, 83-93.

Schacher J.F., Khalil G. M. : Development of Foleyella philistinae Schacher and Khalil, 1967 (Nematoda: Filarioidea) in Culex pipiens molestus with notes on pathology in the Arthropod. J. Parasitol., 1968, 54, 869-878.

Vaucher C., Bain O.: Développement larvaire de Dracunculus doi (Nematoda), parasite d'un serpent malgache, et description de la femelle. Ann. Parasitol. Hum. Comp., 1973, 48, 91-104. 


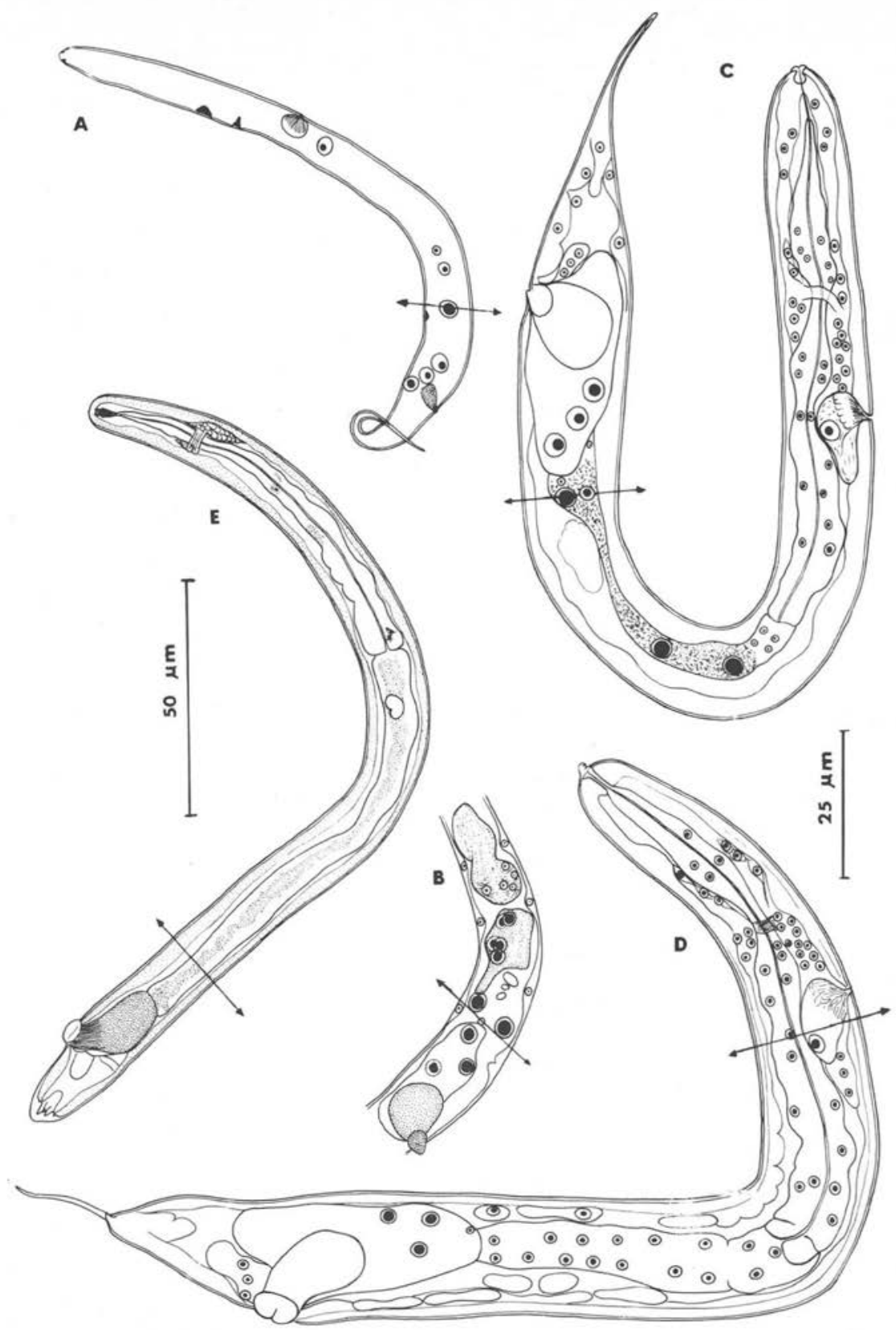

Fig. 1. Morphologie des larves de $D$. (M.) dessetae correspondant aux coupes représentées (les flèches indiquent le niveau de ces coupes). A : stade 1 à 3 jours : cellule $R_{1}$ non divisée et début d'apparition de l'ébauche intestinale ( 2 cellules); B : stade I de 5 jours : premières divisions de la $\mathrm{R}_{1}$, ébauches rectale, intestinale et œsophagienne formées. C : stade I de 6 jours au début de la mue I : cordon digestif mis en place; D : larve de 9 jours au stade II ; E : larve de 11 jours au début de la mue II (d'après Bain, 1974; l'âge indiqué pour les larves correspond au développement chez Aedes aegypti, à $27^{\circ}$ ). 

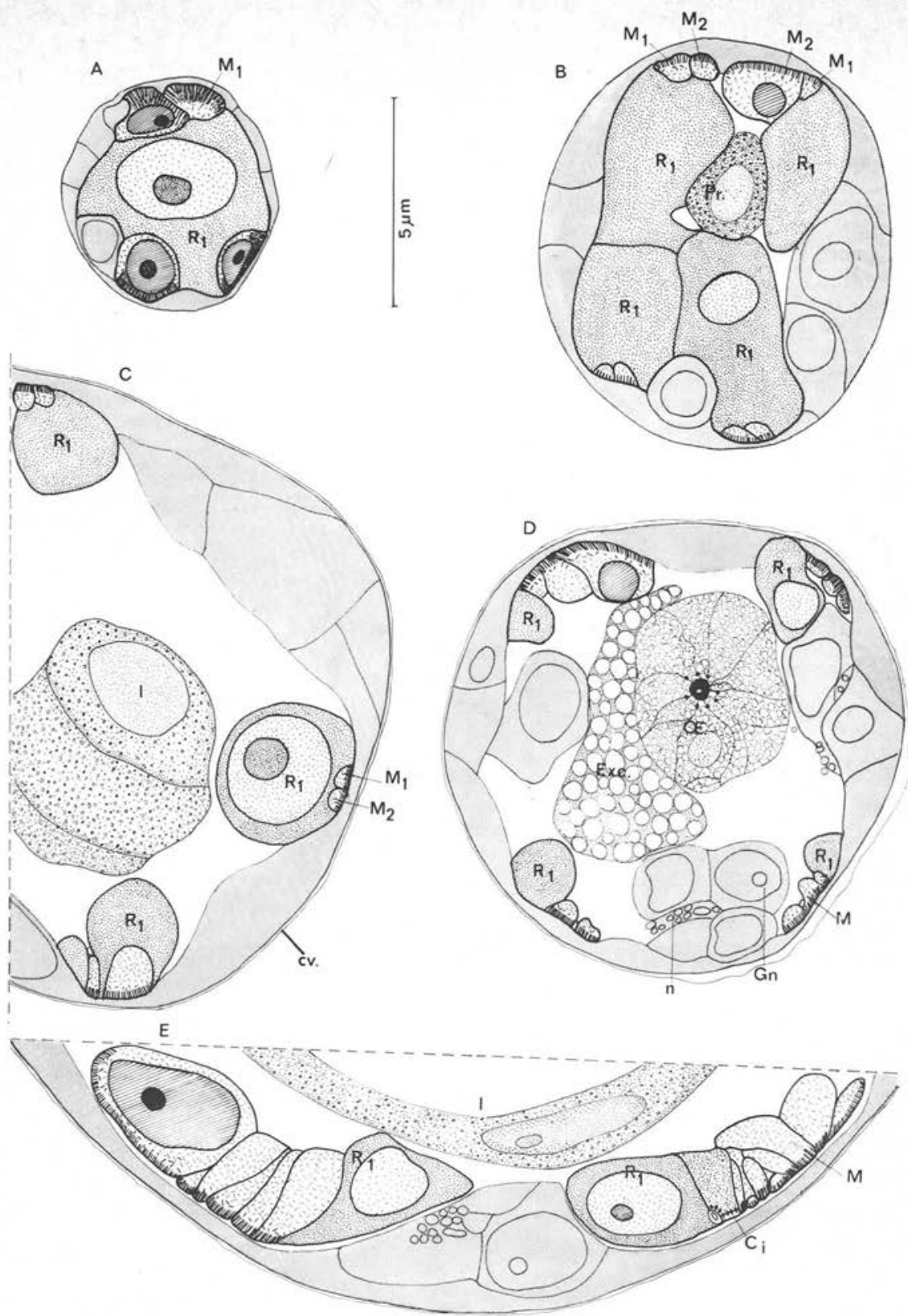

Fig. 2. Evolution de la cellule $\mathrm{R}_{1}$ et de la musculature ; représentation schématique des coupes transversales ultrafines. A : larve de 3 jours: cellule $R_{1}$ non divisée et cellules musculaires primordiales $\mathrm{M}_{1}$ (une par champ). B : larve de 5 jours, au niveau du prérectum ; les premières divisions de la $R_{1}$ ont donné dans chaque champ une cellule embryonnaire $\left(R_{1}\right)$ et une nouvelle cellule musculaire $\left(\mathrm{M}_{2}\right)$ accolée à la cellule musculaire primordiale $\left(\mathrm{M}_{1}\right)$. C : larve de 6 jours, au niveau de l'extrémité postérieure de l'intestin ; D : larve de 9 jours, au niveau de l'œsophage et de la cellule excrétrice: augmentation du nombre des cellules musculaires. $\mathrm{E}$ : larve de 11 jours, au niveau de l'intestin : présence d'une cellule intermédiaire (Ci) entre les cellules de type $R_{1}$ et les cellules musculaires. [L'hypoderme et les éléments nerveux sont en grisé ; les cellules musculaires ont une base striée, haute à 3 jours, plus basse ensuite quand les cellules musculaires sont peu contractiles; da cellule $R_{1}$ et ses descendants non contractiles sont ponctués; la cellule intermédiaire est ponctuée et a des croix à sa base ; éch., $5 \mu \mathrm{m}]$. 


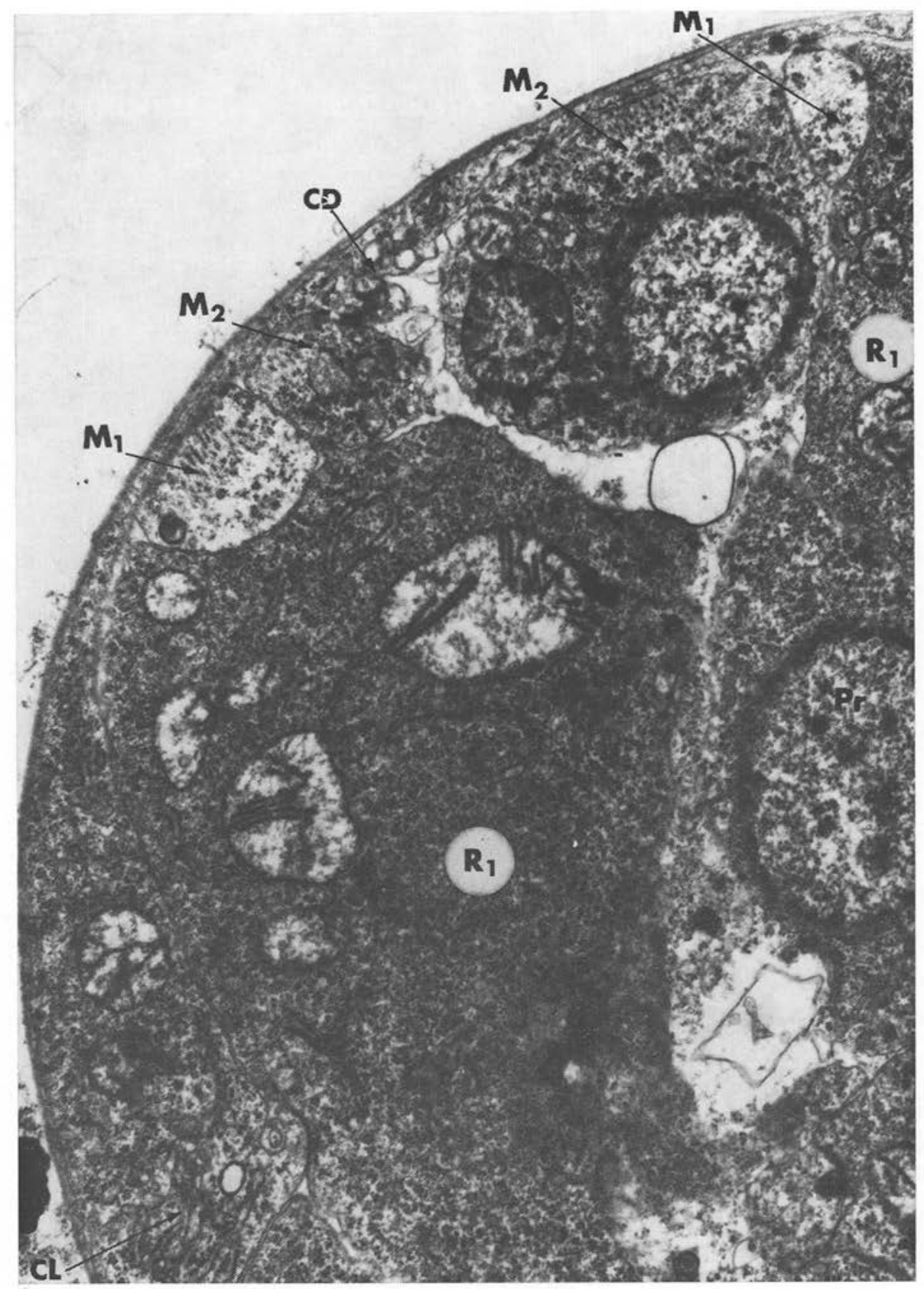

Fig. 3. Deux champs latérodorsaux correspondant à la figure $2 \mathrm{~B}\left(5^{\mathrm{e}}\right.$ jour $)$ : les cellules musculaires primordiales $M_{1}$, plus claires, sont en cours de dédifférenciation ( $~ .28 .000$ ). 


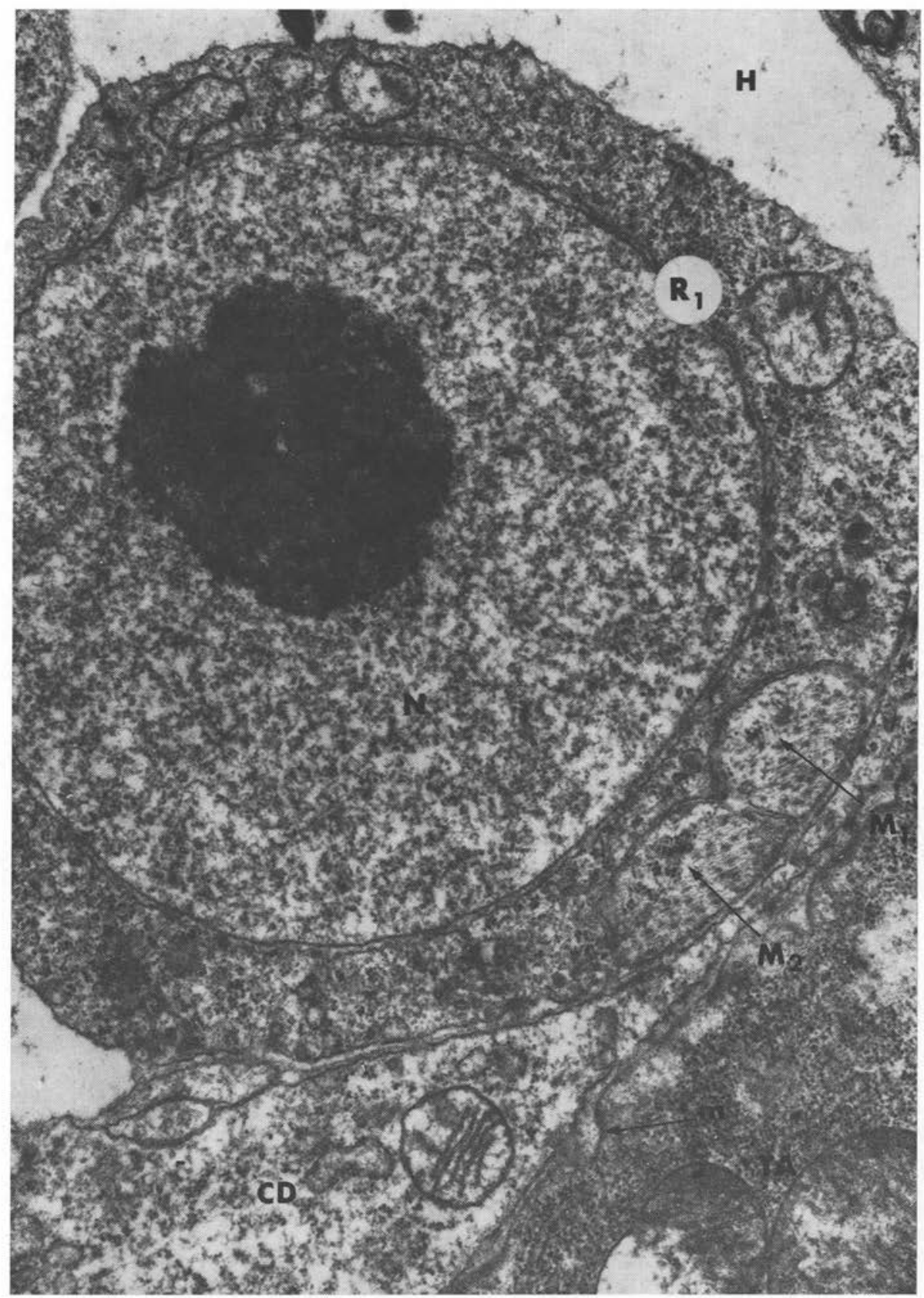

Fig. 4. Un des champs subdmédians de la figure $2 \mathrm{C}\left(6^{e}\right.$ jour $)$ : les cellules musculaires $\mathrm{M}_{1}$ et $\mathrm{M}_{2}$ sont maintenant identiques ( $\times$ 40.000). 


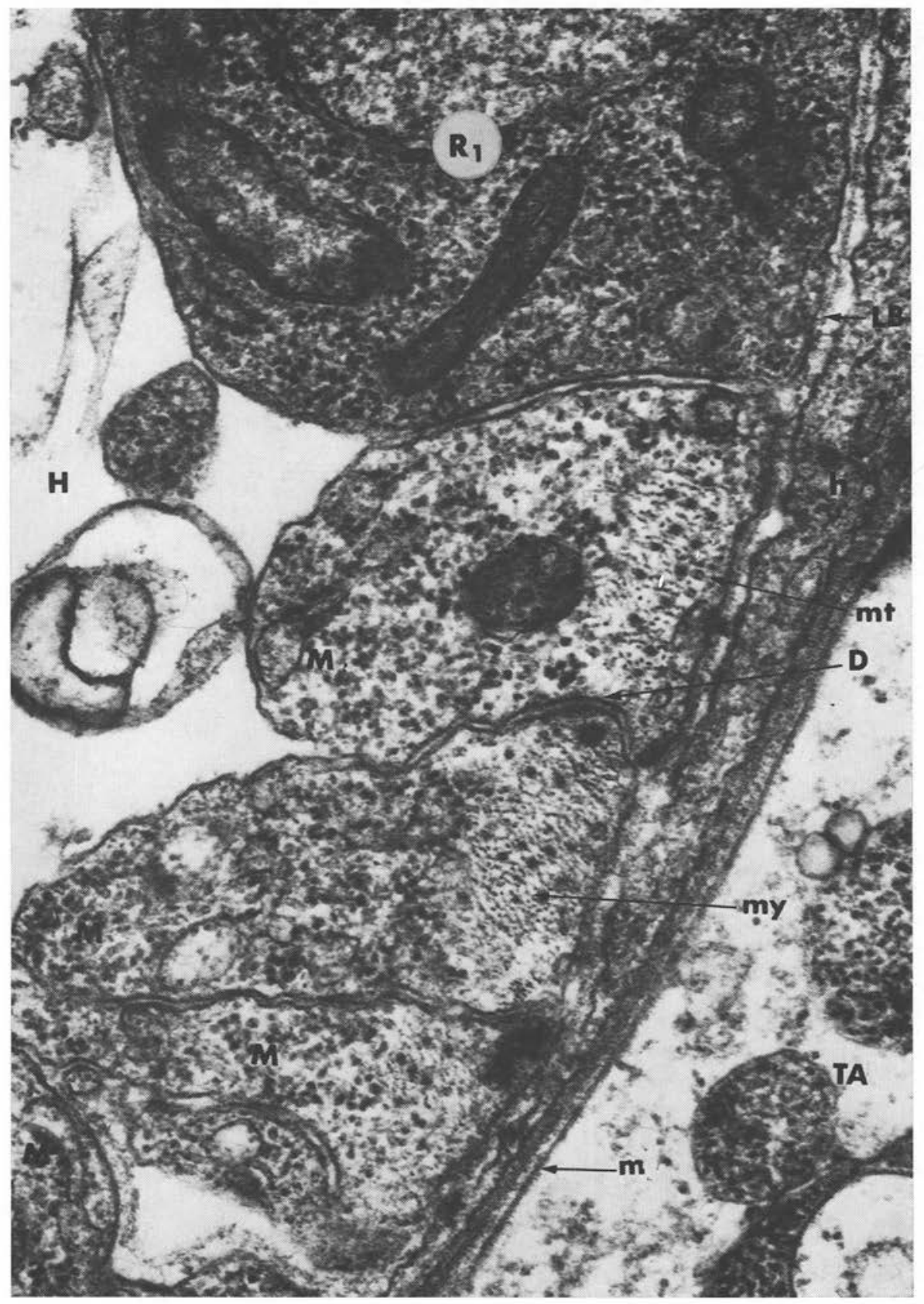

Fig. 5. Un champ submédian de la figure $2 \mathrm{D}$ ( $9^{\circ}$ jour) : la cellule de type $\mathrm{R}_{1}$ et les 4 cellules musculaires $\mathrm{M}$ forment un ensemble, nettement séparé de l'ectoderme par une lame basale $(\times 84.000)$. 


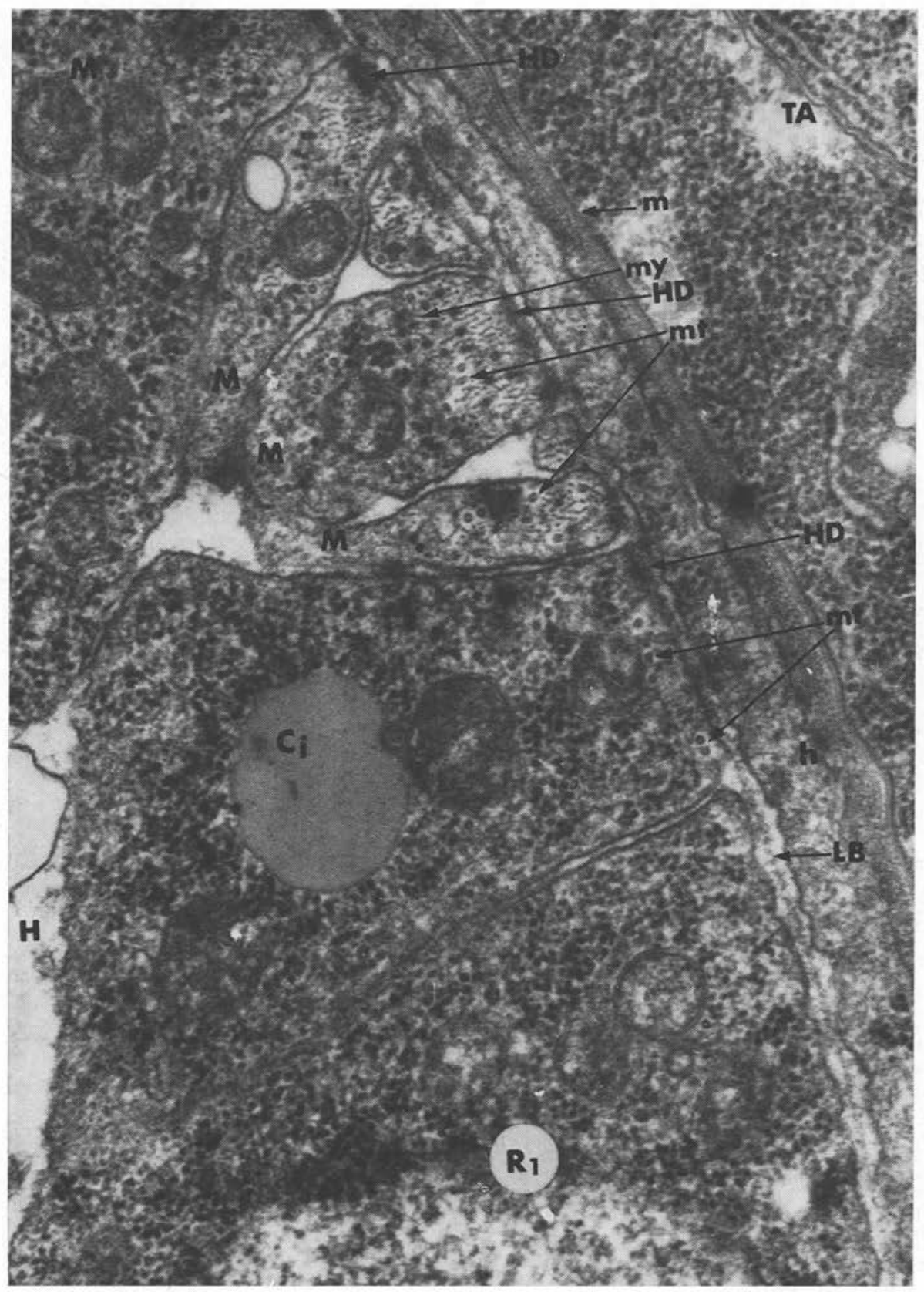

Fig. 6. Un champ submédian de la figure $2 \mathrm{E}\left(11^{\mathrm{e}}\right.$ jour) : il comprend une cellule de type $\mathrm{R}_{1}$, une cellule intermédiaire $(\mathrm{Ci})$ et des cellules musculaires; ces deux derniers types sont fixés à l'hypoderme par des hemidesmosomes $(\times 76.000)$. 


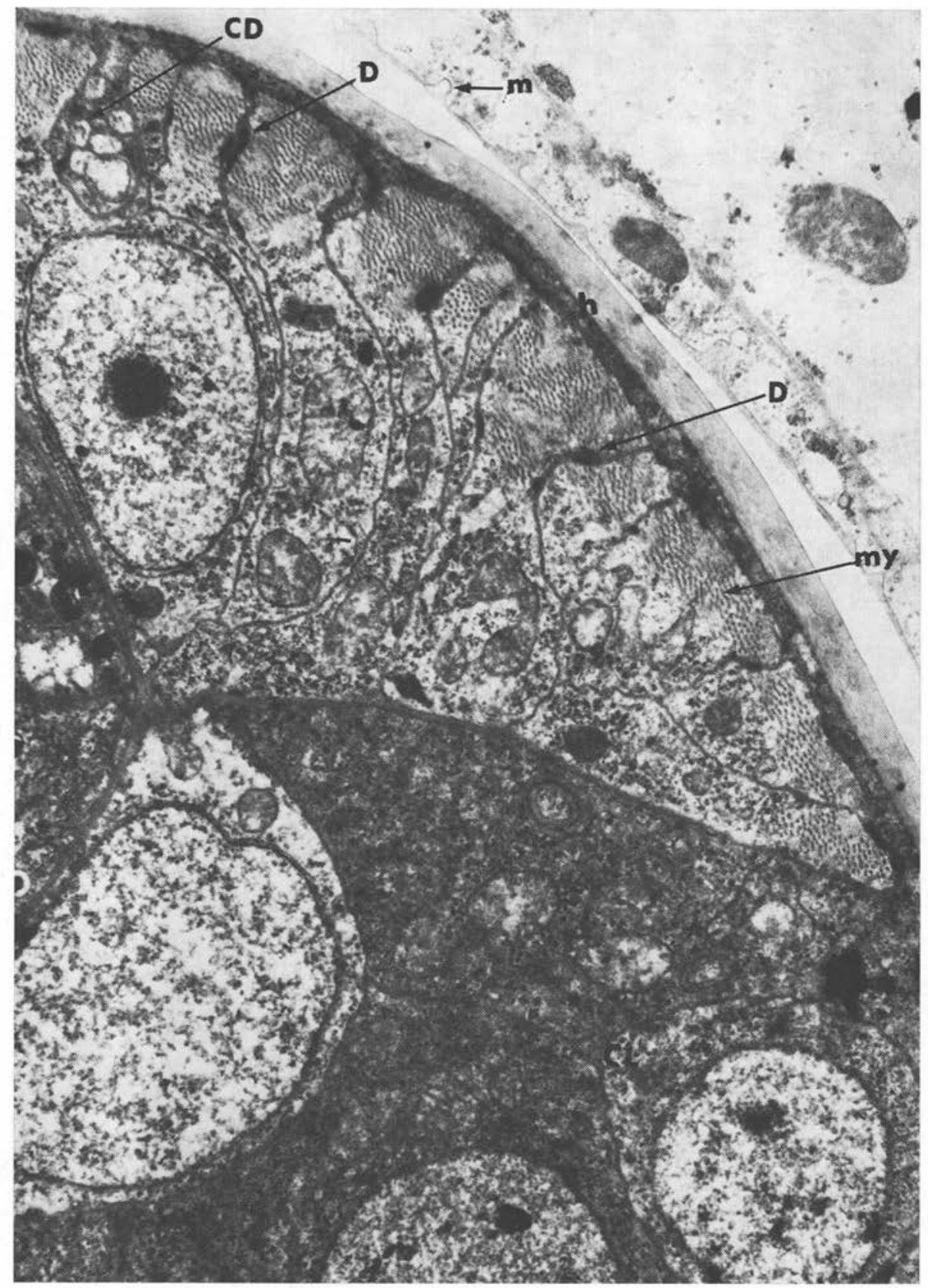

Fig. 7. Un champ submédian d'une larve de 14 jours dans la mue II : toutes les cellules du champ (11) sont musculaires, à partie contractile développée $(\times 20.000)$. 